\title{
MARKOV PARTITIONS FOR EXPANDING MAPS OF THE CIRCLE
}

\author{
MATTHEW STAFFORD
}

\begin{abstract}
We study Markov partitions for orientation-preserving expanding maps of the circle whose rectangles are connected. Up to a reordering of basis elements, the class of induced matrices arising for such partitions is characterized. Then the study focuses on the subclass of partitions for which each boundary set is a periodic orbit. We show that, if the boundary orbit of a partition is well-distributed, the partition and its symmetries can be constructed. An accompanying result is concerned with double covers of the circle only. It says that, for a given period, all partitions bounded by ill-distributed orbits have the same induced matrix.
\end{abstract}

\section{INTRODUCTION}

The aim of this paper is to classify Markov partitions by looking at their induced matrices. For linear endomorphisms, the eigenvector associated with the dominant eigenvalue of an induced matrix has a geometric interpretation. Namely, its entries are the lengths of the rectangles for the partition. This is important for partitions with connected rectangles; it means that a partition can be reconstructed from its induced matrix if we are also provided with the order of the rectangles as we proceed around the circle from a suitable starting point. It follows from work of Shub in [Sh] that the properties of interest here generalize from linear endomorphisms to all expanding maps.

The importance of Markov partitions as a tool was revealed in the study of basic sets for Axiom A diffeomorphisms. Bowen gives a very good exposition of the subject in that context in [B]. In this study, our model for the circle is $[0,1]$ with endpoints identified.

Definition 1.1. A $C^{1}$ map $f: S^{1} \rightarrow S^{1}$ is expanding if $\left|f^{\prime}(x)\right|>1$ for all $x \in S^{1}$.

Definition 1.2. The finite collection $R_{1}, R_{2}, \ldots, R_{p}$ of subsets of $S^{1}$ form a Markov partition for the expanding map $f$ if the following four conditions are satisfied:

Received by the editors February 2, 1989 and, in revised form, December 8, 1989. Presented at the October 21, 1989 AMS Meeting at Stevens Institute of Technology, Hoboken, New Jersey.

1980 Mathematics Subject Classification (1985 Revision). Primary 58F15; Secondary 54H20, $58 \mathrm{~F} 11$. 
(1) For all $i, j$, each point of $\operatorname{int}\left(R_{j}\right)$ has the same number of inverse images in $R_{i}$ under $f$. This is called the Markov condition and is the only requirement involving the map $f$.

(2) $R_{1} \cup R_{2} \cup \cdots \cup R_{p}=S^{1}$.

(3) The interiors of the $R_{i}$ 's are pairwise disjoint.

(4) For every $i, \operatorname{cl}\left(\operatorname{int}\left(R_{i}\right)\right)=R_{i}$.

The $R_{i}$ 's are called the rectangles for the partition.

A Markov partition is connected if each of its rectangles is connected.

The boundary set for a Markov partition is the set of all points which are contained in more than one rectangle.

Definition 1.3. Let $R_{1}, R_{2}, \ldots, R_{p}$ be the rectangles for some Markov partition. For all $i, j$ define $m_{i j}$ to be the nonnegative integer such that each point in $\operatorname{int}\left(R_{j}\right)$ has exactly $m_{i j}$ preimages in $R_{i}$. This gives a $p \times p$ matrix $M=\left(m_{i j}\right)$ which is called the induced matrix or transition matrix for the partition.

In this paper, our attention will be focused on orientation-preserving expanding maps. The discussion in $\S \S 2$ and 3 is completely dependent on this restriction.

It is easy to see that the boundary set for a Markov partition must be an invariant set for the map. The simplest sort of invariant set is a single periodic orbit. The theorem we are about to state says something about Markov partitions whose boundary sets are of this simplest type. A symmetry of a Markov partition is a spin or reflection which has the same induced matrix. A more careful definition of this term will be given later.

Theorem A. Suppose that two connected Markov partitions for an orientationpreserving expanding map of $S^{1}$ induce the same matrix, and that each partition is bounded by a single periodic orbit. Suppose further that the induced matrix is a 0-1 matrix and that every row has at least one 0 . Then the partitions must be symmetries of one another.

This theorem is given as item 4.2 in the exposition. We will proceed toward it as follows. The rest of the introduction is taken up with defining terminology and proving general facts about Markov partitions. In so doing, we pay particular attention to the class of expanding maps which stretch $S^{1}$ uniformly.

In the next section the form of the induced matrix for a connected partition is described, subject to an ordering condition on the rectangles. We give a theorem on constructing a connected partition if given a matrix in this form. The third section is concerned with partitions whose boundary orbits are badly distributed. These are in a sense the opposites of those partitions covered by Theorem A. The last section of the paper is chiefly concerned with proving Theorem A. Most of the material in this paper was developed as part of a thesis project at Northwestern University. The author would like to thank John Franks for his ideas and encouragement connected with this work. 
Definition 1.4. Let $f: X \rightarrow X$ and $g: Y \rightarrow Y$ be maps of topological spaces. Then $f$ and $g$ are topologically conjugate if there exists a homeomorphism $h: X \rightarrow Y$ such that $h \circ f=g \circ h$.

Definition 1.5. For $n \geq 2$ the (orientation-preserving) $n$-fold linear endomorphism of $S^{1}$ is given by $f(x)=n x(\bmod 1)$. For $n=2$ this map is called the doubling map.

Remark 1.6. The $n$-fold linear endomorphism $f$ stretches every interval by a factor of $n$ and is uniformly $n$-to-one onto $S^{1}$.

The iterate $f^{p}$ is the map $x \mapsto n^{p} x(\bmod 1)$. It is easy to show that $f$ fixes $x \in S^{1}$ iff $x$ is of the form $m /(n-1), m$ an integer. It follows that $f^{p}$ fixes all points of the form $m /\left(n^{p}-1\right), m$ an integer.

Lemma 1.7. Let $f$ be the map $x \mapsto n x(\bmod 1)$. Let $p \geq 1,[a, b]$ an arbitrary interval in $S^{1}$. Then

$$
f^{p}[a, b]=\text { all of } S^{1} k \text { times }+\left[f^{p}(a), f^{p}(b)\right] a(k+1) \text { th time } .
$$

The $k$ in the above formula is the integer part of $n^{p} \cdot \operatorname{length}[a, b]$.

Proof. The integer part of $n^{p}$. length $[a, b]$ is also the largest integer $k$ such that $\frac{k}{n^{p}} \leq$ length $[a, b]$. Write $[a, b]$ as $\left[a, a+\frac{k}{n^{p}}\right) \cup\left[a+\frac{k}{n^{p}}, b\right]$. Since $f^{p}\left(a+\frac{k}{n^{p}}\right)=$ $f^{p}(a)$, we conclude that $f^{p}$ maps the first subinterval $k$-to-1 onto $S^{1}$ and maps the second subinterval 1-to-1 onto $\left[f^{p}(a), f^{p}(b)\right]$.

Remark 1.8. Proposition 6 on p. 186 of [Sh] says that every expanding map of $S^{1}$ is topologically conjugate to a linear endomorphism (of the same degree.) Thus any orientation-preserving expanding map of degree $n$ has exactly $n-1$ fixed points.

Theorem 1.9. Let $f$ be an orientation-preserving, expanding map of $S^{1}$ of degree $n$. The group of homeomorphisms of $S^{1}$ which commute with $f$ has order $2(n-1)$.

If $f$ is the $n$-fold linear endomorphism then the group is generated by the rotation $x \mapsto x+1 /(n-1)(\bmod 1)$ and the reflection $x \mapsto 1-x$.

Proof. The identity and the map $m \mapsto-m$ are the only isomorphisms of $\mathbb{Z}$, the fundamental group of $S^{1}$. Both of these isomorphisms commute with $f_{\sharp}: \mathbb{Z} \rightarrow \mathbb{Z}$ (which is multiplication by $n$ ).

The theorem is a corollary of Theorem 5, p. 183 of [Sh]. In effect, that result says that, once we select $x, y \in S^{1}$ which are fixed by $f$ and an isomorphism $i$ of $\mathbb{Z}$ which commutes with $f_{\#}$, there is a unique homeomorphism $\theta$ of $S^{1}$ which commutes with $f$, takes $x$ to $y$, and induces the map $i$ on the fundamental group. The choice of the isomorphism $i$ determines whether the resulting homeomorphism will preserve or reverse orientation.

Definition 1.10. A square matrix $M$ is irreducible and aperiodic if there exists a $k$ for which every entry in $M^{k}$ is strictly positive. 
The next theorem is a standard result from linear algebra; a thorough treatment is found in [Se] beginning on p. 3 .

Theorem 1.11 (Perron-Frobenius). Let $M$ be a nonnegative, irreducible and aperiodic matrix. Then there is a nonnegative eigenvalue, called the Perron value, which is the unique eigenvalue of largest modulus. The Perron value is a simple eigenvalue.

Further, there exist left and right eigenvectors for $M$ corresponding to the Perron value in which each entry is strictly positive. No other eigenvalue has associated to it an eigenvector with all entries nonnegative.

Proposition 1.12. Let $f$ be the $n$-fold linear endomorphism. Let $R_{1}, R_{2}, \ldots$, $R_{p}$ be a Markov partition for $f$ with induced matrix $M$. Then $M$ has the following properties:

(1) The ith row of $M$ describes the image of rectangle $R_{i}$; the jth column of $M$ describes the pre-images of rectangle $R_{j}$.

(2) Each column of $M$ must sum to $n$.

(3) $R_{1}, R_{2}, \ldots, R_{p}$ is also a Markov partition for $f^{q}: S^{1} \mapsto S^{1}$, any $q \geq$ 1. Further, its induced matrix is $M^{q}$.

(4) $M$ is irreducible and aperiodic.

(5) Let $x$ be the column vector whose ith entry is length $\left(R_{i}\right)$. Then $M x=$ $n x$. The stretching factor $n$ is therefore a simple eigenvalue and the unique eigenvalue of largest modulus.

(6) Suppose $g$ is an expanding map of degree $n$ and $\theta$ is a homeomorphism of $S^{1}$ for which $g \circ \theta=\theta \circ f$. Then $\theta\left(R_{1}\right), \theta\left(R_{2}\right), \ldots, \theta\left(R_{p}\right)$ form a Markov partition for $g$ and this new partition also has induced matrix $M$.

Most of these assertions are evident without proof. We remark that (4) relies on the requirement that each rectangle for a Markov partition has nonempty interior; this also establishes that the vector $x$ of property (5) has all entries strictly positive.

To finish with property (5), suppose $M$ is a $0-1$ matrix. The $i$ th entry of $M x$ is just $\sum_{R_{j} \subset f\left(R_{i}\right)}$ length $\left(R_{j}\right)$. This summation is clearly the length of $f\left(R_{i}\right)$, which in turn is $n \cdot$ length $\left(R_{i}\right)$ because $f$ is a rigid stretching. If $M$ contains entries larger than 1 , the summation must be adjusted to include multiplicity. This verifies that $M x=n x$. Therefore $n$ is the Perron value for $M$.

It now makes sense to focus our study on linear endomorphisms. Coupled with Remark 1.8, property (6) above says that exactly the same induced matrices occur for a linear endomorphism as for any expanding map of the same degree and orientation. Therefore properties (1)-(4) of Proposition 1.12 hold for expanding maps in general. But a topological conjugacy may distort the lengths of the rectangles. Thus in (5) we retain the fact that the degree of the map is the Perron value but lose the geometric meaning of the associated eigenvector for nonlinear expanding maps. 
The major results of this paper will be stated as generally as possible. In supporting lemmas, we will typically work with linear endomorphisms, as lengths of rectangles will often come into play. Parts (5) and (6) of the above proposition motivate the next two definitions.

Definition 1.13. Let $M$ be the induced matrix for a Markov partition for a linear endomorphism. The length eigenvector for $M$ is defined to be the right Perron vector of $M$ with entries normalized to sum to 1 .

Definition 1.14. Let $G$ be the group of homeomorphisms of $S^{1}$ which commute with an expanding map $f$, and let $\theta \in G$. Suppose $\mathscr{P}$ is a Markov partition for $f$ with rectangles $R_{1}, R_{2}, \ldots, R_{p}$. Then $\theta(\mathscr{P})$ is defined to be the Markov partition whose rectangles are $\theta\left(R_{1}\right), \theta\left(R_{2}\right), \ldots, \theta\left(R_{p}\right)$.

The set of symmetries of $\mathscr{P}$ is defined to be $\{\theta(\mathscr{P}) \mid \theta \in G\}$.

Definition 1.15. Let $M$ be a $p \times p$ matrix with nonnegative integer entries.

(1) The graph associated to $M, G(M)$, is a directed graph with vertex set $V=\{1,2, \ldots, p\}$. For any pair $i, j$ of vertices, the edge set $E$ contains $m_{i j}$ distinct edges from vertex $i$ to vertex $j$.

(2) We define maps $o, t: E \rightarrow V$ by

$o(e) \equiv$ originating vertex of edge $e$ and

$t(e) \equiv$ terminating vertex of edge $e$.

(3) The relation $\rightarrow$ between elements of $E$ is defined by $e \rightarrow f$ iff $t(e)=$ $o(f)$.

(4) The one-sided shift of finite type $\sum_{M}$ is defined to be that subset of $\prod_{i=0}^{\infty} E$ for which

$$
\bar{e}=e_{0} e_{1} e_{2} \cdots \in \sum_{M} \text { iff } e_{i} \rightarrow e_{i+1} \quad \text { for all } i \geq 0 .
$$

One can think of $\sum_{M}$ as the space of all infinite paths in $G(M)$. We topologize $\sum_{M}$ by starting with the discrete topology on $E$, moving to the product topology on $\prod_{i=0}^{\infty} E$, and restricting to $\sum_{M}$.

The (left) shift map $\sigma: \sum_{M} \mapsto \sum_{M}$ is given by $\sigma\left(e_{0} e_{1} e_{2} \cdots\right)=e_{1} e_{2} e_{3} \cdots$. We remark that $\sigma$ is continuous.

Definition 1.6. Let $M$ be the induced matrix for a Markov partition. The partition is topologically generating if, for every sequence $\bar{e} \in \sum_{M}$,

$$
\bigcap_{i=0}^{\infty} f^{-i}\left(R_{o\left(e_{i}\right)}\right) \text { contains at most one point . }
$$

Definition 1.17. Let $R_{1}, R_{2}, \ldots, R_{p}$ and $S_{1}, S_{2}, \ldots, S_{p}$ be the rectangles for the Markov partitions $\mathscr{P}$ and $\mathscr{Q}$, respectively. Then $\mathscr{Q}$ is a refinement of $\mathscr{P}$ if each rectangle $S_{i}$ of $\mathscr{Q}$ is contained in some rectangle $R_{j}$ of $\mathscr{P}$.

Lemma 1.18. Any refinement of a topologically generating partition is also topologically generating.

Proof. The lemma is obvious from the definitions of the concepts involved. 


\section{CONNECTED PARTITIONS}

Throughout this section, $f$ is the endomorphism $x \mapsto n x(\bmod 1)$ unless otherwise specified.

Lemma 2.1. For a connected partition, the following are equivalent:

(i) the partition is topologically generating.

(ii) all rectangles have length $<1 / n$.

(iii) every entry in the induced matrix is either 0 or 1 and there is at least one 0 in each row.

Proof. That (i) $\Rightarrow$ (ii) is shown by contrapositive. If $[a, b]$ is a rectangle of length $\geq 1 / n$, then $a+1 / n \in[a, b]$ and therefore has exactly the same "itinerary" as $a$. For a rectangle of length exactly $1 / n$, this ambiguity occurs only at the endpoints.

That (ii) $\Rightarrow$ (iii) is clear; in particular, a rectangle of length equal to $1 / n$ would give rise to a row of all 1's in the induced matrix $M$.

For (iii) $\Rightarrow$ (i), we consider sequences of edges from the graph $G(M)$. These will be written in the form $e_{0} e_{1} \cdots e_{k}$, where $e_{i} \rightarrow e_{i+1}$ for all $i$. Induction on $k$ can be used to show that, for every such sequence,

$$
R_{o\left(e_{0}\right)} \cap f^{-1}\left(R_{o\left(e_{1}\right)}\right) \cap f^{-2}\left(R_{o\left(e_{2}\right)}\right) \cap \cdots \cap f^{-k}\left(R_{o\left(e_{k}\right)}\right)
$$

is nonempty, connected, and of length at most $n^{-k}$. The key is that (iii) tells us each rectangle maps 1-to- 1 onto its image .

Compactness then allows the conclusion that, for each $\bar{e} \in \sum_{M}$, the infinite intersection $\bigcap_{i=0}^{\infty} f^{-i}\left(R_{o\left(e_{i}\right)}\right)$ contains exactly one point.

Remark 2.2. Let $R$ be a connected rectangle of some Markov partition for $f$. The intermediate value theorem implies that

$$
R \text { contains a fixed point for } f \Leftrightarrow f(R) \supset R \text {. }
$$

Definition 2.3. For a connected partition, the canonical ordering for the rectangles is given by beginning at the fixed point $0 \in S^{1}$ and proceeding counterclockwise. This ordering can be thought of as a choice of ordered basis for $M$, the induced matrix.

Lemma 2.4. Let $R_{1}, R_{2}, \ldots, R_{p}$ be the rectangles of a connected partition in canonical order. Let $v$ be a row vector of dimension $p$, and suppose each entry in $v$ is one of the integers $s, t$. Define the collections

$$
\begin{aligned}
& C_{s}=\left\{R_{i} \mid \text { ith entry of } v \text { is } s\right\} \text { and } \\
& C_{t}=\left\{R_{j} \mid j \text { th entry of } v \text { is } t\right\} .
\end{aligned}
$$

then the following are equivalent:

(i) The rectangles in $C_{s}$ form a connected union.

(ii) The rectangles in $C_{t}$ form a connected union. 
(iii) The s's and t's in $v$ each form a single contiguous block (wrap-around is allowed).

Proof. The lemma is obvious from the definition of canonical order.

Definition 2.5. Let $M$ be the induced matrix for a connected, topologically generating partition with the canonical ordering of basis elements. The $i$ th row of $M$ will be denoted by $m_{i}$. The symbols $\alpha(i)$ and $\omega(i)$ will denote the positions of the first and last 1 's in $m_{i}$, respectively.

An example serves to illustrate the effect of wrap-around. Suppose $m_{i}=$ $(1,1,0,0,1,1)$. Then $\alpha(i)=5$ and $\omega(i)=2$. In other words, the block of 1 's starts in the fifth position and wraps around through the second position.

Proposition 2.6. Let $f$ be the map $x \mapsto n x$ (mod 1). Suppose the rectangles for a connected, topologically generating partition have been listed in canonical order. $M$ will denote the induced matrix with basis elements in this order and $B$ will denote the boundary set. Then $M$ has the following properties:

(1) $M$ is irreducible and aperiodic. Further, $\sum m_{i}=\bar{n}$, where $\bar{n}$ is the row vector of all $n$ 's.

(2) For all $i, m_{i}$ consists of a single contiguous block of 1 's and a single contiguous block of 0 's, allowing for wrap-around. Thus, in each row, there is at least one 0 and at least one 1.

(3) For $i=1,2, \ldots, p-1, \alpha(i+1)=\omega(i)(\bmod p)+1$. Also, $\alpha(1)=\omega(p)$ $(\bmod p)+1$.

(4) If $m_{i i}=1$, then some other entry in the ith row is also 1.

(5) If $0 \in B, \alpha(1)=1, \omega(1)>1, \alpha(p)<p$, and $\omega(p)=p$. If $0 \notin B, 1<\omega(1)<\alpha(1)$.

(6) $\operatorname{tr}(M)=(n-1)+$ \# of fixed points in $B$.

(7) For all $q$, each row of $M^{q}$ has at most two entries. If there are two entries in a given row, they differ by 1 and are again arranged in contiguous blocks.

Proof. Property (1) restates part of Proposition 1.12. The contiguity of the blocks in property (2) comes from Lemma 2.4. The rest follows from Lemma 2.1 .

Property (3) says that the block of 1's in $m_{i+1}$ is just to the right of the block of 1 's in $m_{i}$ for $i<p$. This is forced by the canonical ordering. In fact, $R_{i}$ and $R_{i+1}$ share an endpoint (as do $R_{p}$ and $R_{1}$ ), so their images must share an endpoint.

Coupled with the fact from (1) about the column sums, we find that the blocks of 1's migrate to the right, wrap around, and migrate to the right a total of $n$ times.

Properties (4) and (5) are clear from Remark 2.2 and the fact that $f$ is expanding.

To prove (6), we first note that each rectangle is too short to contain more than one fixed point. So it is clear that a fixed point $x$ contributes 2 to $\operatorname{tr}(M)$ 
if $x \in B$ and 1 to $\operatorname{tr}(M)$ if $x \notin B$. Now sum over the $n-1$ fixed points of $f$.

Property (7) is a straight application of Lemmas 1.7 and 2.4. The former describes the image of a connected set under $f^{q}$ and the latter guarantees contiguity of the blocks.

Definition 2.7. A matrix satisfying properties (1)-(5) of Proposition 2.6 will be called a connected matrix.

Lemma 2.8. ("DA" operation) Suppose $M$ is the matrix induced by a topologically generating, connected partition for which 0 is not a member of the boundary set $B$. The rectangles and basis elements should be in the canonical order.

Then $B \cup\{0\}$ is invariant and can thus be taken as the boundary set of a new connected partition. This partition is also topologically generating. If $M^{\prime}$ is the induced matrix for the new partition, then

$$
\begin{aligned}
M^{\prime}= & {\left[\begin{array}{cc}
m_{1}^{\prime} & 0 \\
m_{2} & m_{21} \\
m_{3} & m_{31} \\
\vdots & \vdots \\
m_{p} & m_{p 1} \\
m_{1}^{\prime \prime} & 1
\end{array}\right] } \\
\text { where } m_{1}^{\prime}= & (1,1, \ldots 1,0,0, \ldots 0) \\
\uparrow & \omega(1) \text { position } \\
\text { and } m_{1}^{\prime \prime}= & (0,0, \ldots 0,1,1, \ldots 1) \text { sum to } m_{1} . \\
& \begin{array}{c}
\uparrow \\
\end{array}
\end{aligned}
$$

Conversely, let $B^{\prime}$ be the boundary set for a connected partition with induced matrix $M^{\prime}$. Suppose $0 \in B^{\prime}$ but no inverse image of 0 (other than 0 itself) is in $B^{\prime}$. Then $B^{\prime}-\{0\}$ is invariant.

Let $M$ be the induced matrix for the connected partition bounded by $B^{\prime}-\{0\}$. Then $M$ is gotten from $M^{\prime}$ by adding the last row of $M^{\prime}$ to the first and truncating the last row and last column of the result.

In both cases, $\sum_{M^{\prime}}$ and $\sum_{M}$ are not topologically conjugate.

This is an easy lemma which we will not prove (save verifying the last claim in the upcoming remark.)

Remark 2.9. (i) The $M$ that we start with in a "DA" operation is a connected matrix for which $\alpha(1)>1$. The operation can be performed on such a matrix even if we do not know what partition it represents. The result will clearly be a connected matrix for which $\alpha(1)=1$.

(ii) It has been stated that the "DA" operation preserves the property of being topologically generating. The inverse operation does not always do so. 
(iii) In his DA construction, Smale performs a surgery on an Anosov diffeomorphism in a way such that one fixed point is replaced by a pair of fixed points (see [Sm, p. 788]). This is the inspiration for the name of the operation just described. To clarify the similarity of the two settings, the effect of the "DA" operation on the graph of $M$ will be described.

The vertices for $G(M)$ will be labelled $1,2, \ldots, p$. To construct $G\left(M^{\prime}\right)$, we first make a copy of vertices 2 through $p$ and of each edge which originates and terminates among these vertices. The vertex labelled 1 in $G(M)$ will be replaced by two vertices labelled $1^{\prime}$ and $1^{\prime \prime}$ in the new graph.

Note that the vertex labelled 1 has a loop on it, i.e. there is an edge $e$ in $G(M)$ such that $o(e)=t(e)=1$. This loop is duplicated in the sense of putting a loop on each of the two new vertices. However, there will be no edge from $1^{\prime}$ to $1^{\prime \prime}$ or from $1^{\prime \prime}$ to $1^{\prime}$.

Incoming nonloops to vertex 1 are duplicated. Specifically, suppose $o(g) \neq 1$ and $t(g)=1$ for $g$ in $G(M)$. Then we will draw edges $g^{\prime}$ from $o(g)$ to $1^{\prime}$ and $g^{\prime \prime}$ from $o(g)$ to $1^{\prime \prime}$ in $G\left(M^{\prime}\right)$.

Outgoing nonloops from vertex 1 are split. In other words, suppose that $o(g)=1$ and $t(g) \neq 1$ for $g$ in $G(M)$. Then the new graph will have an edge $g^{\prime}$ from $1^{\prime}$ to $t(g)$ or $g^{\prime \prime}$ from $1^{\prime \prime}$ to $t(g)$. Note that we do not draw in both edges.

Now every type of edge in $G(M)$ has been examined. Since the number of loops in $G\left(M^{\prime}\right)$ is one more than that of $G(M)$, it is clear that $\sum_{M^{\prime}}$ has one more fixed point than $\sum_{M}$. This verifies the claim in Lemma 2.8 that the two shifts cannot be topologically conjugate, as any conjugacy would have to map fixed points to fixed points.

Proposition 2.10. Let $M$ be a square matrix and let $f$ be an expanding, orientation-preserving map of degree $n$.

Then there is a connected, topologically generating partition for $f$ which induces $M$ iff $M$ is a connected matrix with column sum $n$ (up to a reordering of basis elements).

If $f$ is a linear endomorphism, this partition can be taken so that the canonical order of the rectangles coincides with the order of basis elements for $M$. In this case the connected partition which induces $M$ and satisfies this ordering condition is unique. Lastly, 0 is a boundary point for the partition iff $\alpha(1)=1$.

Proof. In the first section of this paper, the observation was made that a topological conjugacy between expanding maps establishes a correspondence between their partitions. We add here that this correspondence respects the properties of connectedness and of being topologically generating. So we can assume $f$ is the $n$-fold linear endomorphism.

The forward implication now restates Proposition 2.6. For the converse, we first treat the case where $\alpha(1)=1$. If $M$ is a $p \times p$ matrix satisfying conditions (1)-(5) of Proposition 2.6, we first note that $\overline{1}$, the row vector of all 1's, is a left Perron vector for $M$ and that the Perron value for $M$ is $n$. Let $x$ be a 
right Perron vector for $M$, normalized so that its entries sum to 1 . The strategy we follow now is an obvious one: think of $x$ as a length eigenvector and use the circular order that $M$ itself seems to suggest. A long but unimaginative calculation (which is not reproduced here) will verify that this approach works. The uniqueness in the statement follows because, once a canonical order is chosen for the rectangles, everything else is forced by the knowledge of how long each rectangle must be.

Now we move on to the case where $\alpha(1)>1$. Heeding part (i) of Remark 2.9, we perform a "DA" operation on the matrix $M$, yielding a connected matrix $M^{\prime}$ for which $\alpha(1)=1$. Then, using the construction cited in the first part of this proof, a partition is found which induces $M^{\prime}$. Performing the inverse of the "DA" operation on this new partition yields a partition which induces $M$. This completes the proof.

Definition 2.11. The circular order for a connected partition is just the knowledge, for each rectangle, of which two rectangles are adjacent to it.

In other words, to say that the circular order is given does not imply that the rectangles are listed starting at a fixed point or that we know whether they are listed in clockwise or counterclockwise order.

Corollary 2.12. Let $f$ be orientation-preserving and expanding. Suppose we are given the induced matrix for a connected, topologically generating partition for $f$, as well as the circular order of the rectangles. Then the partition and its symmetries can be constructed.

Proof. A topological conjugacy between expanding maps takes symmetries to symmetries and does not disturb the circular order. So we can again assume the map is a linear endomorphism.

First the rectangles may have to be reordered. Start with a rectangle $R$ containing a fixed point (the corresponding diagonal element of the induced matrix $M$ will be nonzero). The image of $R$ contains one or both of the rectangles adjacent to $R$ because $R$ contains a fixed point and $f$ is expanding. The rectangle which is listed next should be both adjacent to $R$ and contained in $f(R)$. Then we finish the list of rectangles by continuing through the circular order.

The resulting ordering is the canonical order for one of the symmetries of the original partition. Using a similarity transformation, reorder the basis elements for $M$ in the corresponding way to get a new matrix $M^{\prime}$. It follows that $M^{\prime}$ is a connected matrix. Proposition 2.10 now insures that there is a unique way to construct a connected partition with the given canonical order. This completes the proof.

Remark 2.13. The above lemma does not imply that we know which partition is the original and which are its symmetries. However, it leads one to hope that, under certain conditions, a partition and its symmetries could be constructed from information in the induced matrix. The next proposition is the first of two affirmative results along these lines. 
Proposition 2.14. Let $f$ be the doubling map. Suppose we have two distinct connected, topologically generating partitions with the same induced matrix. Suppose that 0 is a boundary point for both partitions, and that $1 / 2$ is a boundary point for neither.

Then the two partitions are reflections of one another about the point $1 / 2$.

Proof. Let $M$ be the common induced matrix, and let $\mathscr{P}$ be either of the partitions. It suffices to show that we can recover the circular order for $\mathscr{P}$ or its reflection purely from information in $M$ and its powers. We will do this by finding, for each rectangle $R_{i}$, a specific dyadic rational which lies in $R_{i}$. Then the rectangles can be ordered merely by sorting these sample points.

There are exactly two rectangles containing the fixed point $0 \in S^{1}$. These are easily identified by locating the nonzero diagonal elements of $M$; let $R$ be one of these rectangles. Assume for the time being that $R$ is of the form $[0, a] . R$ maps across itself and has exactly one other pre-image $R^{\prime}$. Clearly, $1 / 2 \in R^{\prime}$. Further, every rectangle other than $R^{\prime}$ is a subset of $[0,1 / 2)$ or of $(1 / 2,1]$.

Next, let $T$ and $T^{\prime}$ be the two rectangles which map across $R^{\prime}$. So $1 / 4 \in T$ and $3 / 4 \in T^{\prime}$ or vice versa. We claim that there is an $m \geq 0$ for which $f^{m}(R)$ contains one of $T, T^{\prime}$ but not both. In fact, the appropriate row of $M^{m}$ will reveal this, and suffices to distinguish between the two possibilities. Remember that $R$ is the designated rectangle which contains the fixed point.

If the claim were not true, then there would exist an $m$ for which

$$
\begin{aligned}
& f^{m}(R) \text { covers neither } T \text { nor } T^{\prime}, \quad \text { and } \\
& f^{m+1}(R) \text { covers both } T \text { and } T^{\prime} .
\end{aligned}
$$

That would mean that $f^{m}(a)<1 / 4$ and $f^{m+1}(a)>3 / 4$, where $a$ is the clockwise endpoint of $R$. This is a contradiction, so the claim is verified.

Suppose now that we have named $T$ and $T^{\prime}$ so that $f^{m}(R)$ contains $T$ but not $T^{\prime}$. Then $1 / 4 \in T$ and $3 / 4 \in T^{\prime}$. Now we repeat the process. If $U$ and $U^{\prime}$ map to $T$, then $1 / 8 \in U$ and $5 / 8 \in U^{\prime}$ or vice versa. We can tell which of these occurs by finding an $m \geq 0$ such that $f^{m}(R)$ contains one of $U, U^{\prime}$, but not both. Similarly, by looking at the rectangles which map to $T^{\prime}$, one can tell which rectangle contains $3 / 8$ and which contains $7 / 8$. This process can be continued until we have found at least one point in each rectangle. So the circular order for the partition $\mathscr{P}$ has been reconstructed.

Earlier in the proof, we made the assumption that $R$ was of the form $[0, a]$. If $R$ is of the form $[b, 1]$ instead, then its reflection $\bar{R}$ is the set $[0,1-b]$. So the above algorithm will yield the circular order for $\overline{\mathscr{P}}$, the reflection of $\mathscr{P}$. This completes the proof of proposition 2.14 .

\section{ORBITS WITH ONLY ONE PHASE DIFFERENCE}

Throughout this section, $f$ is the endomorphism $x \mapsto n x(\bmod 1)$ unless otherwise specified. 
Definition 3.1. Let $x$ and $y$ be members of a periodic orbit. The phase difference from $x$ to $y$ is the smallest nonnegative integer $k$ such that $f^{k}(x)=y$.

Suppose $R=[x, y]$ is a rectangle for a partition bounded by a periodic orbit. (This just means that every boundary point for the partition lies along the same periodic orbit.) Then the phase difference for $R$ is defined to be the phase difference from the clockwise endpoint $x$ to the counterclockwise endpoint $y$.

Definition 3.2. A connected Markov partition with boundary set $B$ is boundaryinvertible if $\left.f\right|_{B}$ is invertible.

Example 3.3. The connected partition bounded by the orbit of $m /\left(n^{p}-1\right)$, where $p>2$ and $m$ is any integer such that $1 \leq m \leq n-1$.

Since $n^{p-1} m \leq n^{p-1}(n-1)<n^{p}-1$, the members of the orbit have numerators $m, n m, n^{2} m, \ldots, n^{p-1} m$. Note that the counterclockwise ordering of these elements coincides with the order in which they are traversed by $f$. The length of the rectangle $\left[m /\left(n^{p}-1\right), n m /\left(n^{p}-1\right)\right]$ is clearly $(n-1) m /\left(n^{p}-1\right)$.

This partition is the prototype for the following result. Its induced matrix and length eigenvector are given there.

Proposition 3.4. Let $f$ be the map $x \mapsto n x(\bmod 1)$. Let $p>2$ and $m$ be any integer such that $1 \leq m \leq n-1$. Then, for a partition bounded by a period $p$ orbit, the following are equivalent:

(1) The partition has a rectangle of length $\frac{m(n-1)}{n^{p}-1}$.

(2) Up to a reordering of symbols, the induced matrix takes the form

$$
\left[\begin{array}{ccccccc}
0 & 1 & 0 & 0 & \ldots & 0 & 0 \\
0 & 0 & 1 & 0 & \ldots & 0 & 0 \\
0 & 0 & 0 & 1 & \ldots & 0 & 0 \\
\vdots & \vdots & \vdots & \vdots & \ddots & \vdots & \vdots \\
0 & 0 & 0 & 0 & \ldots & 1 & 0 \\
m-1 & m-1 & m-1 & m-1 & \ldots & m-1 & m \\
n-m+1 & n-m & n-m & n-m & \ldots & n-m & n-m
\end{array}\right]
$$

Proof. One can check that the length eigenvector for the matrix given in (2) is $\left(m(n-1), n m(n-1), n^{2} m(n-1), \ldots, n^{p-2} m(n-1), n^{p}-1-m\left(n^{p-1}-1\right)\right)^{T}$.

Denominators have been cleared; in other words, the entries of this vector are normalized so that they sum to $n^{p}-1$ instead of 1 . The fact that $m \leq n-1$ guarantees that the last entry is positive; this suffices to show that $(2) \Rightarrow(1)$.

For the converse, let $[a, b]$ be the rectangle of length $m(n-1) /\left(n^{p}-1\right)$. Then each of the sets $[a, b],[f(a), f(b)], \ldots,\left[f^{p-1}(a), f^{p-1}(b)\right]$ is a union of rectangles. Let these sets be called $J_{0}, J_{1}, \ldots, J_{p-1}$ respectively. The $J_{i}$ 's cover $S^{1}$. (For, each member of the boundary orbit is the clockwise endpoint of exactly one of them.) 
If we show that the lengths of the $J_{i}$ 's sum to 1 , it will follow that they are the rectangles for the partition. For $i=1,2, \ldots, p-2, n^{i} m(n-1)<n^{p}-1$ (recall that $m<n)$. So $f^{i}\left(J_{0}\right)$ is not all of $S^{1}$ and $J_{i}=f^{i}\left(J_{0}\right)$. For later reference, we also note that $f\left(J_{i-1}\right)=J_{i}$.

To compute length $\left(J_{p-1}\right)$, multiply length $\left(J_{p-2}\right)$ by $n$ and remove the integer part. It will simplify the calculations if we work with numerators only and then mod out by $n^{p}-1$. One finds that

$$
n \text {. length }\left(J_{p-2}\right)=(m-1)\left(n^{p}-1\right)+\left[n^{p}-1-\left(n^{p-1}-1\right) m\right] .
$$

This verifies that the length of $J_{p-1}$ is as expected, and that the $J_{i}$ 's are the rectangles. It also confirms that the image of $J_{p-2}$ is all of $S^{1}(m-1)$ times $+J_{p-1}$ an $m$ th time. In other words, the penultimate row of the induced matrix is

$$
(m-1, m-1, m-1, \ldots, m-1, m) \text {. }
$$

Most of the work of verifying the form of the induced matrix has already been done. We already know that the first $p-2$ rows of the induced matrix have 1 's on the first superdiagonal and 0's elsewhere. The last row is now determined because each column sum is $n$.

It is clear from the above proof that each rectangle for the partition in question has the same phase difference. For the partition of Example 3.3, this phase difference is 1 . Notice that we have abandoned the canonical order in favor of starting with the shortest rectangle and proceeding through its successive "images". This is because the canonical order is not recoverable from the matrix of Proposition 3.4. In fact, if $k$ is the phase difference for each rectangle, the rectangles are seen in the order $J_{0}, J_{k}, J_{2 k}(\bmod p)$ etc. So the order depends on the phase difference.

Proposition 3.5. Suppose a Markov partition is boundary-invertible, connected and has a rectangle of length $>(n-1) / n$. Then the induced matrix has a bottom row of $(n, n-1, n-1, \ldots, n-1), 1$ 's along the first superdiagonal, and 0 's elsewhere. Further, the boundary set is a single periodic orbit.

Proof. Let $R=[a, b]$ be the rectangle of length $>(n-1) / n$. Then $f(R)=$ all of $S^{1}(n-1)$ times $+[f(a), f(b)]$ an $n$th time.

Claim:

(1) $[f(a), f(b)]$ is a single rectangle.

(2) The image of every rectangle other than $R$ is a single rectangle.

For (1), suppose $c \in(f(a), f(b))$ is a boundary point. All $n$ inverse images of $c$ are contained in $R$, contradicting boundary-invertibility.

To prove (2), the reasoning is the same. Let $R^{\prime}$ be some rectangle other than $R$. If $f\left(R^{\prime}\right)$ has a boundary point $c$ in its interior, then $n-1$ preimages of $c$ lie in $\operatorname{int}(R)$ and the $n$th lies in $\operatorname{int}\left(R^{\prime}\right)$. Again this contradicts boundary-invertibility and proves the claim.

The rectangle $[f(a), f(b)]$ will be called $R_{1}$ in what follows. 
Claim: $f^{p-1}\left(R_{1}\right)=R$.

To show this, let $k$ be the smallest number such that $f^{k}\left(R_{1}\right) \supseteq R$. For $j<k, f^{j}\left(R_{1}\right)$ is a single rectangle other than $R$. (This is verified inductively using part (2) of the first claim.) So $f^{k}\left(R_{1}\right)=f\left(f^{k-1}(R)\right)$ is a single rectangle and is thus equal to $R$.

Now we have demonstrated that $R_{1}, f\left(R_{1}\right), f^{2}\left(R_{1}\right), \ldots, f^{k}\left(R_{1}\right)$ is a list of rectangles. To verify the claim, we must show that $k=p-1$. Since $f$ stretches each interval, no rectangle can appear twice and $k \leq p-1$.

We will show that no rectangle for the given partition is omitted from the list; it follows that $k=p-1$. Suppose that $R^{\prime}$ were a rectangle not listed. Let $j$ be the smallest number such that $f^{j}\left(R^{\prime}\right)$ is a rectangle in the list; assume without loss of generality that $j=1$.

If $f\left(R^{\prime}\right)=R_{1}$, then $R_{1}$ is covered $n$ times by $f(R)$ and an $(n+1)$ th time by $f\left(R^{\prime}\right)$, a contradiction. Similarly, $f\left(R^{\prime}\right)$ cannot be any other rectangle in the list. This proves the claim that $f^{p-1}\left(R_{1}\right)=R$. With the rectangles in the order $R_{1}, f\left(R_{1}\right), f^{2}\left(R_{1}\right), \ldots, f^{p-1}\left(R_{1}\right)$, we have concurrently verified that the induced matrix is as claimed.

Lastly, we must prove that the boundary set for the partition in question is a single periodic orbit. To do so, let $R$ and $R^{\prime}$ be any two rectangles. Then either $f^{j}(R)=R^{\prime}$ for some $j$ or $f^{j}\left(R^{\prime}\right)=R$ for some $j$. This stems directly from the form of the induced matrix. Assume the latter holds.

Let $x$ and $y$ be the clockwise endpoints of $R$ and $R^{\prime}$, respectively. Then $f^{j}(x)=y$, as $f$ is orientation-preserving. As every boundary point is the clockwise endpoint of some rectangle, all boundary points must lie on the same periodic orbit.

Next we address the question of how many partitions have a rectangle of a given length. The following result shows that the solution is number-theoretic.

Proposition 3.6. Let $f$ be the map $x \mapsto n x(\bmod 1)$. Let $p$ and $0<k<p$ be fixed. Then

$$
\exists q \text { such that } f^{k}\left(\frac{q}{n^{p}-1}\right)=\frac{q+m}{n^{p}-1} \Leftrightarrow\left(n^{k}-1, n^{p}-1\right) \mid m .
$$

Suppose $q_{0}$ is a solution and let $d=\left(n^{k}-1, n^{p}-1\right)$. Then there are exactly $d$ mutually incongruent solutions $\left(\bmod n^{p}-1\right)$ given by

$$
q_{0}, q_{0}+\left(n^{p}-1\right) / d, q_{0}+2\left(n^{p}-1\right) / d, \ldots, q_{0}+(d-1)\left(n^{p}-1\right) / d .
$$

Proof. Recall that $f^{k}$ is multiplication by $n^{k}$. After clearing denominators, one finds that solutions to the above equation are in 1-to-1 correspondence with solutions to the equivalence

$$
\left(n^{k}-1\right) q \equiv m \quad\left(\bmod n^{p}-1\right) .
$$

The solutions to such equivalences are well known from elementary number theory. 
Corollary 3.7. The length of any rectangle for a partition bounded by a period $p$ orbit is a multiple of $(n-1) /\left(n^{p}-1\right)$.

Proof. Clearly, $n-1$ is a divisor of $n^{k}-1$ for all $k \geq 1$.

Remark 3.8. Let $\mathscr{P}$ be the partition bounded by $\mathscr{O}(x)$, a period $p$ orbit. Suppose $\mathscr{P}$ has a rectangle of length $m(n-1) /\left(n^{p}-1\right)$ for $m \leq n-1$. Let $k$ be the phase difference for the rectangles of this partition. (We remarked after Proposition 3.4 that all of the phase differences are the same.) Proposition 3.6 implies that the partition bounded by $\mathscr{O}(x+r)$ has a rectangle of the same length, where $r=1 /\left(n^{p}-1, n^{k}-1\right)$. This new partition is a counterclockwise rotation of $\mathscr{P}$. Note that, if $\left(n^{p}-1, n^{k}-1\right)=n-1$, the new partition is one of the symmetries of $\mathscr{P}$.

In the case where the degree of $f$ is 2 , the preceding results fit together nicely. The following theorem records a few facts which are peculiar to that setting.

Theorem 3.9. Let $f$ be an orientation-preserving, expanding map of degree 2 . For $p>1$, the number of partitions which are boundary-invertible, not topologically generating, and have $p$ rectangles is the same as the number of solutions to $\left(2^{k}-1,2^{p}-1\right)=1$ with $0<k<p$. Each of these partitions is bounded by a single period $p$ orbit, and each induces the $p \times p$ matrix with 1 's on the first superdiagonal, a bottom row of $(2,1, \ldots, 1)$, and 0 's elsewhere.

Proof. It suffices to give a proof for the doubling map $x \mapsto 2 x(\bmod 1)$.

Apply Lemma 2.1 to conclude that any nongenerating partition for the doubling map must have a rectangle of length $\geq 1 / 2$. A rectangle of length exactly $1 / 2$ would contradict boundary-invertibility, as its endpoints would have the same image. Then Proposition 3.5 tells us that the matrix is as claimed, and that the boundary set is just one orbit. Now we know that the partition has a rectangle of length $1 /\left(2^{p}-1\right)$ (Proposition 3.4.)

The proof can be finished by using Proposition 3.6 to find out how many partitions have restangles of length $1 /\left(2^{p}-1\right)$.

Corollary 3.10. Let $f$ be an orientation-preserving, expanding map of degree 2 . Every connected partition for $f$ with more than one boundary orbit is topologically generating. (This does not require that the partition be boundary-invertible.) Proof. Let $\mathscr{P}$ be the given partition and $B$ its boundary set. Then $\mathscr{P}$ is a refinement of the connected partition bounded by

$$
B^{\prime}=\text { the set of all periodic points in } B \text {. }
$$

Apply the above theorem to the new partition.

\section{Generating ORBits}

Definition 4.1. A periodic orbit is generating if the (connected) partition bounded by that orbit is topologically generating. 
It is worth noting that a generating orbit is well distributed in the sense that neighboring members of such an orbit are never too far apart. This section is mainly devoted to proving theorem A of the introduction, which is now restated.

Theorem 4.2. Let $f$ be orientation-preserving and expanding. Suppose two connected Markov partitions for $f$ induce the same matrix, and that each partition is bounded by a single, generating periodic orbit.

Then the partitions must be symmetries of one another. In fact, given the common induced matrix, the partitions and all of their symmetries can be constructed.

It is somewhat surprising that such strong hypotheses are needed to prove a theorem of this sort. The discussion in the previous section provides ample evidence that one cannot drop the requirement that the partitions be topologically generating. Even when the degree of the map is 2, there are instances where many nongenerating partitions induce the same matrix (Theorem 3.9.) Before furnishing a proof of Theorem 4.2, the following example is given to show that some sort of boundary-invertibility assumption is necessary as well.

Example 4.3. We exhibit two topologically generating partitions which induce the same matrix, but for which the circular orders are not analogous. The map in both cases is the doubling map $x \mapsto 2 x(\bmod 1)$.

The rectangles for the first partition are

$$
\begin{gathered}
R_{1}=[33 / 62,1 / 62], \quad R_{2}=[1 / 62,1 / 31], \\
R_{3}=[1 / 31,2 / 31], \quad R_{4}=[2 / 31,4 / 31], \quad R_{5}=[4 / 31,8 / 31], \\
R_{6}=[8 / 31,16 / 31], \quad R_{7}=[16 / 31,33 / 62] .
\end{gathered}
$$

The endpoints with denominator 31 form a period 5 orbit. Clearly, the points with denominator 62 map into this orbit on the first iteration. Here the order of the indices corresponds with the canonical order.

The rectangles for the second partition are

$$
\begin{gathered}
S_{1}=[41 / 62,9 / 62], \quad S_{2}=[9 / 62,5 / 31], \\
S_{3}=[9 / 31,10 / 31], \quad S_{4}=[18 / 31,20 / 31], \quad S_{5}=[5 / 31,9 / 31], \\
S_{6}=[10 / 31,18 / 31], \quad S_{7}=[20 / 31,41 / 62] .
\end{gathered}
$$

Again the boundary set consists of a period 5 orbit along with 2 points which enter the periodic orbit after one iterate.

A routine check will verify that, with the basis elements in the order in which the rectangles are listed, both partitions induce the following matrix. The important thing to notice is that the rectangles for the first partition are listed in canonical order. But for the second partition, the canonical order would be $S_{1}, S_{2}, S_{5}, S_{3}, S_{6}, S_{4}, S_{7}$. So it is clear that the two partitions are not symmetries. 


$$
\left[\begin{array}{lllllll}
1 & 1 & 0 & 1 & 1 & 1 & 1 \\
0 & 0 & 1 & 0 & 0 & 0 & 0 \\
0 & 0 & 0 & 1 & 0 & 0 & 0 \\
0 & 0 & 0 & 0 & 1 & 0 & 0 \\
0 & 0 & 0 & 0 & 0 & 1 & 0 \\
1 & 1 & 0 & 0 & 0 & 0 & 1 \\
0 & 0 & 1 & 0 & 0 & 0 & 0
\end{array}\right]
$$

The proof of Theorem 4.2 requires the following lemma.

Lemma 4.4. Suppose that the induced matrix for the partition bounded by a generating periodic orbit is given. Then knowing that one pair of rectangles is adjacent suffices to determine the circular order. Further, such a pair of adjacent rectangles can always be found.

Proof. First it is shown that one adjacent pair determines the circular order. Let $p \geq 4$ be the period of the boundary orbit. For $p=2,3$ there is nothing to prove.

Suppose it is given that rectangles $R$ and $S$ intersect. For the sake of argument let $x$ be the point of intersection and assume that $x$ is the clockwise endpoint of $S$.

The map $F=\left.f\right|_{\mathscr{O}(x)}$ is invertible. Let $S^{\prime}$ be the unique rectangle such that $f\left(S^{\prime}\right)$ contains $S$ but not $R$. Such a rectangle exists and is unique because $F^{-1}(x)$ is the clockwise endpoint of a unique rectangle and the partition is topologically generating. Similarly, let $R^{\prime}$ be the unique rectangle such that $f\left(R^{\prime}\right)$ contains $R$ but not $S$. It is clear that $R^{\prime} \cap S^{\prime}=F^{-1}(x)$. We emphasize that $R^{\prime}$ and $S^{\prime}$ can be selected using information only from the induced matrix.

By iterating this procedure, we will eventually visit each member of the boundary orbit. At each boundary point, we will learn which two rectangles intersect. This clearly suffices to reconstruct the circular order, finishing the proof of the first statement.

The statement that an adjacent pair of rectangles can be found is harder to prove. The next few lemmas and definitions develop the necessary machinery.

Remark 4.5. Suppose $M$ is induced by a connected partition, and pick $n \geq 1$. Recall property (7) of Proposition 2.6: the $i$ th row of $M^{n}(\bmod 1)$ describes two complementary collections of rectangles. Each collection forms a connected union. If $R_{i}=[a, b]$, these connected components are delineated by $f^{n}(a)$ and $f^{n}(b)$. (If $f^{n}(a)=f^{n}(b)$, just think of one component as all of $S^{1}$ and the other being empty.) In this way, each row of each power of $M$ describes a complementary pair of connected components of $S^{1}$.

Definition 4.6. Let $p>3$ be the number of rectangles for a connected partition. A collection of rectangles which form a connected union is nontrivial if it 
contains $\geq 2$ rectangles and $\leq p-2$ rectangles. (Thus a collection is nontrivial iff the complementary collection is nontrivial.)

Definition 4.7. Let $M$ be the induced matrix for a connected partition. Then $\Gamma=\Gamma_{M}$ is defined to be the collection of nontrivial connected components delineated by all of the powers of $M$.

We remark that the collection $\Gamma$ is closed under complements.

Definition 4.8. The collection $\Gamma$ separates the rectangles $R$ and $S$ if there is a complementary pair of connected components in $\Gamma$ for which $R$ and $S$ are not in the same component.

Lemma 4.9. Let $p>2$. Each partition bounded by a generating period $p$ orbit has at lease two distinct phase differences which do not sum to $p$.

Proof. First we show that there must be at least two phase differences for such a partition. Let $R=[a, b]$ be a rectangle for a given partition and assume to the contrary that every rectangle has phase difference $k$. The interval $[f(a), f(b)]$ also has phase difference $k$ and is thus the unique rectangle with clockwise endpoint $f(a)$. According to Lemma 1.7,

$$
f(R)=\text { all of } S^{1} m \text { times }+[f(a), f(b)] \text { an }(m+1) \text { th time } .
$$

Since the partition is generating, $m$ must be 0 . Therefore the row in the induced matrix $M$ corresponding to $R$ sums to 1 . The same is true of every other row in $M$ for the same reason; thus the sum of all of the entries in $M$ is $p$. This contradicts our knowledge that each column sum is the degree of $f$ (which is at least 2). Therefore there are two rectangles with distinct phase differences.

Now suppose that $k$ and $p-k$ are the only two phase differences for our partition. Then one can find boundary points $a, b$ and $c$ such that $[a, b]$ is a rectangle of phase difference $k$ and $[b, c]$ is a rectangle of phase difference $p-k$. It follows that $f^{p}(a)=c$, contradicting that $a$ has period $p$. This proves the lemma.

Lemma 4.10. Let $M$ be the induced matrix for a partition bounded by a generating period $p$ orbit. Then the associated collection $\Gamma$ of connected components separates every pair of adjacent rectangles for the partition.

Proof. Let $[a, b]$ and $[b, c]$ be adjacent rectangles of phase differences $k$ and $k^{\prime}$, respectively. We prove the case where $k \neq k^{\prime}$ and $2 k \neq p$. The other cases are handled similarly.

By hypothesis, $f^{k}(a)=b$. So $\left[f^{k}(a), f^{k}(b)\right]$ contains $[b, c]$ and $\left[f^{k}(b)\right.$, $\left.f^{k}(a)\right]$ contains $[a, b]$. We want to show that the containment is proper in each case. To do so it suffices to show that $f^{k}(b)$ cannot be $a$ or $c$. If $f^{k}(b)=a$, then $f^{2 k}(a)=a$ and thus $2 k=p$, a contradiction. If $f^{k}(b)=c$, then $k=k^{\prime}$, which has also been ruled out. Thus the row in $M^{k}$ corresponding to $[a, b]$ separates the rectangles $[a, b]$ and $[b, c]$ nontrivially. This finishes the special case. 
Now the proof of Lemma 4.4 can be completed. We need to indicate how to use the previous lemma to establish that a single pair of rectangles is adjacent.

Recall that $\Gamma$ is the collection of nontrivial connected components determined by the powers of the induced matrix. Let $Y=[a, b]$ be a minimal component from $\Gamma$ (in the sense of containing the fewest rectangles). If the number of rectangles in $Y$ is two, these two rectangles are adjacent and the proof is finished. So assume that $Y$ contains more than two rectangles. Minimality insures that the intersection of $Y$ with any other component in $\Gamma$ is connected (unless it is just $\{a, b\}$.) For, if $[c, d]$ were a component in $\Gamma$ whose intersection with $Y$ was disconnected with nonempty interior, the "complementary" component $[d, c]$ would be a proper subset of $Y$.

Let $c \in[a, b]$ be a boundary point such that $[a, c]$ is a union of exactly two rectangles. There is some component $Z$ in $\Gamma$ whose counterclockwise endpoint is $c$. Using minimality again, $[a, c]$ is properly contained in $Z$. Therefore $Z \cap Y$ is a union of two adjacent rectangles. This finishes the proof of Lemma 4.4 .

Proof of Theorem 4.2. Lemma 4.4 says that the circular order for either of the given partitions can be recovered from the common induced matrix. Thus the circular orders are the same. Corollary 2.12 then guarantees that the two partitions are symmetries of one another and the proof is finished.

\section{REFERENCES}

[B] R. Bowen, Equilibrium states and the ergodic theory of Anosov diffeomorphisms, Lecture Notes in Math., vol. 470, Springer-Verlag, Berlin and New York, 1975.

[Se] E. Seneta, Non-negative matrices and Markov chains, 2nd ed., Springer-Verlag, New York, 1981.

[Sh] M. Shub, Endomorphisms of compact differentiable manifolds, Amer. J. Math. 91 (1969), 175-199.

[Sm] S. Smale, Differentiable dynamical systems, Bull. Amer. Math. Soc. 73 (1967), 747-818.

[St] M. Stafford, Markov partitions for the doubling map, Ph.D. Thesis, Northwestern Univ., June 1989.

Institute of Mathematics and Its Applications, University of Minnesota, MinneapoLis, Minnesota 55455

Current address: Austin, Texas 78712

Department of Mathematics, RLM 8100, University of Texas at Austin, 